\title{
TheraBite exercises to treat trismus secondary to head and neck cancer
}

\author{
Jolanda I. Kamstra • Jan L. N. Roodenburg • \\ Carien H. G. Beurskens • Harry Reintsema • \\ Pieter U. Dijkstra
}

Received: 27 July 2012 / Accepted: 19 September 2012 / Published online: 4 October 2012

(C) The Author(s) 2012. This article is published with open access at Springerlink.com

\begin{abstract}
Purpose The aim of this study was to evaluate the effect of TheraBite exercises on mouth opening and to analyze factors influencing this effect in a patient record evaluation.

Methods Effect of exercises with a TheraBite to treat trismus was evaluated in 69 head and neck cancer patients of two university medical centers. Mouth opening was measured as interincisal distance in millimeters. Patient, tumor, and treatment characteristics were analyzed for their relationship with change in mouth opening. Variables univariately associated $(p \leq 0.05)$ with change in mouth opening were entered in a logistic regression analysis as possible predictors for an increase in mouth opening of the smallest detectable difference of $5 \mathrm{~mm}$ or more.

Results Mean initial mouth opening was $22.0 \mathrm{~mm}$ (SD 6.4); mean increase in mouth opening was $5.4 \mathrm{~mm}$ (SD 5.7). "Chemotherapy", "medical center", and "time from oncological treatment to start exercises" were significantly associated with an increase in mouth opening. In the logistic regression analysis, "medical center" $(\beta=1.97)$ and "time from oncological treatment to start exercises" reduced the
\end{abstract}

\author{
J. I. Kamstra $(\bowtie) \cdot$ J. L. N. Roodenburg $\cdot H$. Reintsema \\ P. U. Dijkstra \\ Department of Oral and Maxillofacial Surgery, \\ University Medical Center Groningen, University of Groningen, \\ PO Box 30.001, 9700 RB Groningen, The Netherlands \\ e-mail: j.i.kamstra@umcg.nl \\ C. H. G. Beurskens \\ Department of Physical Therapy, Radboud University Nijmegen \\ Medical Center, Radboud University Nijmegen, \\ PO Box 9.102, 6500 HC Nijmegen, The Netherlands \\ P. U. Dijkstra \\ Department of Rehabilitation, Center for Rehabilitation, \\ University Medical Center Groningen, University of Groningen, \\ PO Box 30.001, 9700 RB Groningen, The Netherlands
}

odds of reaching an increase in mouth opening of $5 \mathrm{~mm}$ or more, $\beta=-0.11$ per month.

Conclusions After TheraBite exercises, mouth opening increased averagely with $5.4 \mathrm{~mm}$. The odds of an increase in mouth opening of $5 \mathrm{~mm}$ or more reduces if the time from oncological treatment to start exercises lengthens, corrected for the effect of medical center.

Keywords Head and neck neoplasms · Trismus · Exercise therapy $\cdot$ Rehabilitation

\section{Introduction}

Trismus, a restricted mouth opening, is defined as a mouth opening of $35 \mathrm{~mm}$ or less [1-3]. It is a well-known complication of cancer in the head and neck region and its treatment. The prevalence of trismus ranges from 5 to $38 \%$ [1]. The consequences of trismus can be serious; it can result in problems with speech, oral hygiene, dental treatment, and oncological follow-up [4-6]. Furthermore, trismus impacts negatively on mandibular functioning and quality of life $[5,7-9]$.

Trismus in head and neck cancer patients is difficult to treat. Many (unusual) stretching techniques have been described in case reports and case series. A sledgehammer, tied to the mandible for 2 min twice a day, resulted in an increase in mouth opening of $18 \mathrm{~mm}$ [10], An orthodontic "clothes pin appliance" inserted between the molars and thereby stretching mouth opening resulted in an increase of $6 \mathrm{~mm}$ [11]. Further, application of a surgical mouthprop, a tapered screw, a screw-type mouth gag, fingers, tongue depressors, an intraoperatively fabricated self-curing bite block, and interarch springs attached between maxillary and mandibular restorations have been described as methods to improve 
mouth opening but without providing information about the actual increase in mouth opening [12-16]. Injection of botulinum toxin in both masseter muscles did not show improvement of mouth opening in head and neck cancer patients [17]. Currently, no standard type of therapy to treat trismus exits [18].

Historical cohort studies provided evidence that conventional exercise therapy (using tongue depressors, fingers, and rubber plugs) has some effect; mean increase in mouth opening of $5.5 \mathrm{~mm}$ [19]. Jaw-stretching devices demonstrated efficacy in improving trismus of head and neck cancer patients in several studies with different designs [18, 20-23].

Exercises with the Dynasplint ${ }^{\circledR}$ Trismus System (DTS) increased mouth opening between the 6.2 and $13.6 \mathrm{~mm}$, as described in a case series, a preliminary report, and in a retrospective study $[18,22,23]$. Exercises with the TheraBite ${ }^{\circledR}$ Jaw Motion Rehabilitation System ${ }^{\mathrm{TM}}$ (TheraBite) increased mouth opening with 13.6 and $10 \mathrm{~mm}$, as described in a randomized controlled trial with short follow-up $(n=21$ with 10 weeks follow-up) and in a pilot study with small sample size ( $n=7$ with $12-48$ weeks follow-up), respectively [20, 21]. However, factors influencing the effect of TheraBite exercises are unknown.

The aim of this study was to evaluate the clinical effect of TheraBite exercise therapy to treat trismus secondary to head and neck cancer and to analyze factors influencing this effect in 69 head and neck cancer patients of two medical centers. It was hypothesized that tumor and oncological treatment characteristics, as well as time from oncological treatment to start exercises influence the effect of TheraBite exercises.

\section{Methods}

All head and neck cancer patients of two university medical centers with trismus, treated with TheraBite exercise therapy between April 2004 and July 2011 were included. Characteristics regarding the cancer and its treatment were obtained from medical records. Patients were referred from the Department Head and Neck Oncology to the Department of Physical Therapy for exercise therapy. Tumor classification was based on the Union for International Cancer Control recommendation (2009) TN classification [24]. Trismus was defined as a mouth opening of $35 \mathrm{~mm}$ or less [1].

The majority of the data were obtained retrospectively from the medical/physical therapeutic records. However, 15 patients had not completed their exercise therapy at the start of this study. Therefore, data of mouth opening of these patients were collected prospectively at follow-up appointments. All of these patients gave written permission for the use of these data for scientific purposes. The institutional review board approved this study.

\section{TheraBite exercises}

Exercises with the TheraBite are patient-controlled. The mouthpieces are placed between the maxilla and mandible. The stretching effects occur when the handle of the TheraBite is squeezed. During their first visit to the Department of Physical Therapy, patients were familiarized with the use of the TheraBite and received exercise instructions. Follow-up appointments were routinely planned every 2 weeks to evaluate possible difficulties encountered during the exercise therapy. Generally, about three instruction sessions were held to inform and motivate the patient. When the patient was comfortable with the exercises, follow-up was planned individually. Patients were instructed that pain during the stretch was normal, but excessive pain should be avoided. Additionally, patients were informed that their teeth or alveolar ridges could become painful because of the pressure. During the TheraBite exercise therapy, patients did not receive concomitant treatments to improve mouth opening.

Exercises were performed according to instructions given by the physical therapist. Patients in the first medical center (Radboud University Nijmegen Medical Center; RUNMC) received the instruction to exercise four sessions each day, with 6-8 repetitions within each session, and hold the stretch for $10-15 \mathrm{~s}$. In the second medical center (University Medical Center Groningen; UMCG), the instructions were to perform the exercises five sessions each day, with 6 repetitions within each session, and hold the stretch for $6 \mathrm{~s}$. Patients were encouraged to continue exercising following this schedule until no further improvement in mouth opening could be achieved.

\section{Measurement of mouth opening}

Mouth opening was measured prior to TheraBite exercises and at regular follow-up appointments by using a slide caliper. In patients with complete frontal dentition, mouth opening was measured as the maximal interincisal distance. In edentulous patients wearing dentures, distance between the incisors of the upper and lower dentures was measured. In edentulous patients not wearing dentures, maximal distance between the two alveolar ridges was measured. In patients with one edentulous and one jaw with frontal dentition wearing dentures, distance between the incisor of denture and the incisor was measured. In patients with one edentulous en one jaw with frontal dentition not wearing dentures, distance between the alveolar ridge and the incisor was measured. 
Statistical analyses

Statistical analyses were performed using SPSS 18.0 for Windows software (SPPS Inc., Chicago, IL, USA). In the univariate analyses, associations between patient, tumor, and treatment characteristics (predictor variables) and the increase in mouth opening of $5 \mathrm{~mm}$ or more (outcome variable) were analyzed by means of Mann-Whitney $U$ (MWU), Kruskal-Wallis, and Spearman's rho $(\rho)$. Variables univariately associated $(p \leq 0.05)$ with the outcome variable "increase in mouth opening of $5 \mathrm{~mm}$ or more" (yes, no) were entered in the multivariate logistic regression analysis (stepwise backward). This increase of $5 \mathrm{~mm}$ or more corresponds with the smallest detectable difference of single measurement of mouth opening in patients with head and neck cancer [25].

The following predictor variables were analyzed regarding their relationship with increase in mouth opening: gender (female, male), squamous cell carcinoma (yes, no), radiotherapy (yes, no), surgery (yes, no), chemotherapy (yes, no), chemoradiation (yes, no), medical center (RUNMC, UMCG), $\mathrm{T}$ classification (T1-T3, T4), tumor site (oral cavity; oro-, naso-, hypopharynx; temporomandibular joint region), age (years), initial mouth opening (millimeters), duration of exercise therapy (months), and time from oncological treatment (counted from last date of the surgery, chemotherapy, radiotherapy, or chemoradiation) to start (TheraBite) exercises (months).

\section{Results}

Between April 2004 and July 2011, 72 patients were provided with a TheraBite. Measurements of mouth opening pre and post exercises were available of 69 patients. Of three patients, no measurement of final mouth opening was available because they deceased due to their cancer during the exercise therapy. They were excluded. Dental status of none of the patients changed between initial measurement and last measurement of mouth opening. Patient and tumor characteristics are presented in Table 1. Mean initial mouth opening was $22.0 \mathrm{~mm}$ (SD 6.4). Mean increase in mouth opening after TheraBite exercises was $5.4 \mathrm{~mm}$ (SD 5.7). In 37 patients $(54 \%)$, mouth opening increased with $5 \mathrm{~mm}$ or more. Of 49 patients $(71 \%)$, the tumor was staged as T4. Of nine patients, TN classification was missing: five of these patients had a recurrence, two patients an osteosarcoma, one patient an adenoid cystic carcinoma, and one patient had a rhabdomyosarcoma 18 years ago (Table 2).

In one patient, the reconstruction plates fractured during TheraBite exercises therapy; this was diagnosed during oncological follow-up by means of an orthopantomogram. The patient was treated with surgery and postoperative
Table 1 Patient characteristics $(n=69)$ and tumor characteristics

\begin{tabular}{|c|c|c|}
\hline Variables & Mean (SD) & $n(\%)$ \\
\hline Female & & $32(46.4)$ \\
\hline Radiotherapy & & $54(78.3)$ \\
\hline Surgery & & $51(73.9)$ \\
\hline Chemotherapy & & $14(20.3)$ \\
\hline Chemoradiation & & $12(17.4)$ \\
\hline \multicolumn{3}{|l|}{ Medical center } \\
\hline RUNMC & & $33(47.8)$ \\
\hline UMCG & & $36(52.2)$ \\
\hline Age in years & $53.9(14.9)$ & \\
\hline Initial mouth opening in $\mathrm{mm}$ & $22.0(6.4)$ & \\
\hline Increase in mouth opening in $\mathrm{mm}$ & $5.4(5.7)$ & \\
\hline Increase of $5 \mathrm{~mm}$ or more & & $37(53.6)$ \\
\hline Duration of exercise therapy in months & $6.3(5.6)$ & \\
\hline $\begin{array}{l}\text { Time from oncological treatment to } \\
\text { start exercises in months }{ }^{\mathrm{a}}\end{array}$ & $15.1(38.7)$ & \\
\hline \multicolumn{3}{|l|}{ Type of tumor } \\
\hline Squamous cell carcinoma & & $49(71.0)$ \\
\hline Salivary gland carcinoma & & $6(8.7)$ \\
\hline Rhabdomyosarcoma & & $4(5.8)$ \\
\hline Osteosarcoma & & $3(4.3)$ \\
\hline Merkel cell carcinoma & & $2(2.9)$ \\
\hline Meningioma & & $1(1.4)$ \\
\hline Chondrosarcoma & & $1(1.4)$ \\
\hline Epidermoid cyst & & $1(1.4)$ \\
\hline Adenoid cystic carcinoma & & $1(1.4)$ \\
\hline Unknown $^{\mathrm{b}}$ & & $1(1.4)$ \\
\hline \multicolumn{3}{|l|}{ Site of tumor } \\
\hline Oral cavity & & $33(47.8)$ \\
\hline Oro-, naso-, hypopharynx & & $28(40.6)$ \\
\hline Temporomandibular joint region & & $8(11.6)$ \\
\hline
\end{tabular}

${ }^{a}$ Some patients started TheraBite exercise therapy before ending radiotherapy

${ }^{\mathrm{b}}$ One patient with a large cell carcinoma was treated with chemotherapy and radiation, no final pathology rapport was present

radiotherapy for a pT4N0 squamous cell carcinoma in the lower jaw. The mandibular reconstruction had been performed with a free vascularized fibula transplant, fixated with titanium plates. During exercises, the patient complained of grating sounds that were initially interpreted as crepitation arising from the temporomandibular joint.

Table 2 TN classification

\begin{tabular}{rrrrr}
\hline & $\mathrm{N} 0$ & $\mathrm{~N} 1$ & $\mathrm{~N} 2$ & $\mathrm{~N} 3$ \\
\hline $\mathrm{T} 1$ & 4 & 0 & 2 & 0 \\
$\mathrm{~T} 2$ & 4 & 4 & 5 & 0 \\
$\mathrm{~T} 3$ & 1 & 0 & 1 & 0 \\
$\mathrm{~T} 4$ & 6 & 4 & 28 & 1 \\
\hline
\end{tabular}


Approximately 6 weeks after the plate fracture was diagnosed, it became clear she was suffering from a large inoperable recurrence. If this patient was excluded from the statistical analysis, mean increase in mouth opening changed to $5.5 \mathrm{~mm}$ (SD 5.7). For further data analyses, her data were excluded.

\section{Results of statistical analyses}

Variables significantly associated with increase in mouth opening were: "chemotherapy" (MWU, $p=0.04)$, "medical center" (MWU, $p=0.01$ ), and "time from oncological treatment to start exercises" $(\rho=-0.516, p<0.01)$ (Table 3). These variables were entered in the logistic regression analysis. If the time between oncological treatment and start exercises lengthens, the odds of obtaining a mouth opening $5 \mathrm{~mm}$ or more reduced, while being treated in the RUNMC increased the odds (Table 4, Fig. 1).

\section{Discussion}

On average, mouth opening improved $5.4 \mathrm{~mm}$ (SD 5.7) after TheraBite exercises. The factors influencing the efficacy of TheraBite exercises were chemotherapy, medical center, and time from oncological treatment and start exercises in the univariate analysis. The influence of medical center on the effect of exercises may be caused by differences in patient population, exercise protocol, or by compliance to the exercise therapy. Also unknown center effects may be responsible for differences between centers. Effects of radiotherapy on increase in mouth opening were not found in this study, probably because that the majority of the patients $(78 \%)$ received radiotherapy.

The results of the logistic regression analysis showed that the odds of reaching an increase in mouth opening of $5 \mathrm{~mm}$ or more reduced if the time from oncological treatment to start exercises lengthened and if patients were treated in the UMCG. Chemotherapy did not significantly influence the odds of reaching an increase in mouth opening of $5 \mathrm{~mm}$ or more in the logistic regression analysis, maybe due to lack of adequate sample size. Thus, our initial hypothesis was only partially confirmed. Clinically, our results indicate that if patients start with the exercises after 1 year after the oncological treatment in the UMCG, the odds ratio of improving mouth opening with $5 \mathrm{~mm}$ of more is 0.27 , $\mathrm{e}^{\left(12^{*}-0.108\right)}$, compared to a person who starts immediately after the oncological treatment (Fig. 1).

If trismus, caused by surgery or radiotherapy and the related fibrosis (scar tissue), exists for a certain period, the restriction may become more resistant to exercise therapy due to maturation of scar tissue [26]. This maturation can explain the reduced of the effect of exercise therapy when the time from oncological treatment to start exercises lengthens. Results of previous studies suggest that TheraBite exercise therapy is superior in increasing mouth opening compared to conventional exercise therapy [20, 21]. Based on those results, head and neck cancer trismus patients were
Table 3 Univariate analyses of independent variables related to increase in mouth opening

\begin{tabular}{llll}
\hline Variables & \multicolumn{2}{l}{ Increase in mouth opening Median (IQR) } & Test, significance \\
\hline Gender & Male & Female & \\
& $3.0(1.5 ; 7.5)$ & $5.5(1.3 ; 9.8)$ & MWU, $p=0.29$ \\
Yes & No & MWU, $p=0.74$ \\
Squamous cell carcinoma & $4.0(2.0 ; 8.0)$ & $5.0(0.0 ; 9.8)$ & MWU, $p=0.75$ \\
Radiotherapy & $5.0(1.0 ; 8.0)$ & $5.0(2.0 ; 7.0)$ & MWU, $p=0.10$ \\
Surgery & $6.0(1.0 ; 9.0)$ & $3.0(1.5 ; 5.5)$ & MWU, $p=0.04$ \\
Chemotherapy & $2.0(0.0 ; 4.3)$ & $6.0(2.0 ; 9.0)$ & MWU, $p=0.81$ \\
Chemoradiation & $4.5(2.3 ; 6.8)$ & $5.0(1.0 ; 8.5)$ & MWU, $p=0.01$ \\
Medical center & RUNMC & UMCG & \\
T classification & $7.0(3.0 ; 8.5)$ & $2.0(0.0 ; 6.0)$ & MWU, $p=0.38$ \\
& T1-T3 & T4 & KW, $p=0.32$ \\
Tumor site & $6.0(2.0 ; 8.0)$ & $4.0(0.0 ; 8.0)$ & \\
& Oral cavity $6.0(1.0 ; 8.5)$ & $\rho=0.166, p=0.17$ \\
& Oro-, naso-, hypopharynx $2.5(1.3 ; 7.0)$ & $\rho=0.014, p=0.91$ \\
Age & Temporomandibular joint region $8.0(1.5 ; 10.5)$ & $\rho=-0.100, p=0.42$ \\
Initial mouth opening & & & $\rho=-0.516, p<0.01$ \\
Duration of exercise therapy & & & \\
Time from oncological treatment to & & & \\
start exercises & & & \\
\hline
\end{tabular}


Table 4 Multivariate logistic regression analysis (stepwise backward) with an increase in mouth opening of $5 \mathrm{~mm}$ or more as outcome variable

\begin{tabular}{lrrrrrrr}
\hline & $\beta$ & Standard error $\beta$ & $P$ value & Odds ratio & \multicolumn{2}{c}{$95 \%$ confidence interval } \\
\cline { 3 - 7 } & & & & & Lower & Upper \\
\hline Medical center & & & & & & \\
Time from oncological treatment to start exercises & -0.108 & 0.04 & 0.005 & 7.094 & 1.83 & 27.51 \\
Constant & 0.197 & 0.42 & 0.003 & 0.897 & 0.84 & 0.96 \\
\hline
\end{tabular}

${ }^{\mathrm{a}}$ Reference center is UMCG

provided with a TheraBite routinely in our centers. However, the results of those studies could not be confirmed in our study. The superior effects of exercises with a TheraBite in those studies might also be related to the sample size, population characteristics, or time from oncological treatment to start exercise.

It is possible that the effect of TheraBite exercises in this study is smaller than those found in 1993 by Buchbinder et al. due to confounding by indication. This type of confounding indicates that patients were referred only if they had a severely restricted mouth opening. When comparing the mean initial mouth opening and standard deviations of our patients (21.8 mm, SD 6.6) and those of Buchbinder et al. $(21.3 \mathrm{~mm}, \mathrm{SD} 1.7)$, it can be seen that only standard deviations differ between the groups. In our group, a larger variation in initial mouth opening was present [20].

In a pilot study of Cohen et al. in 2005, the mean increase in mouth opening after TheraBite exercises was $10 \mathrm{~mm}$

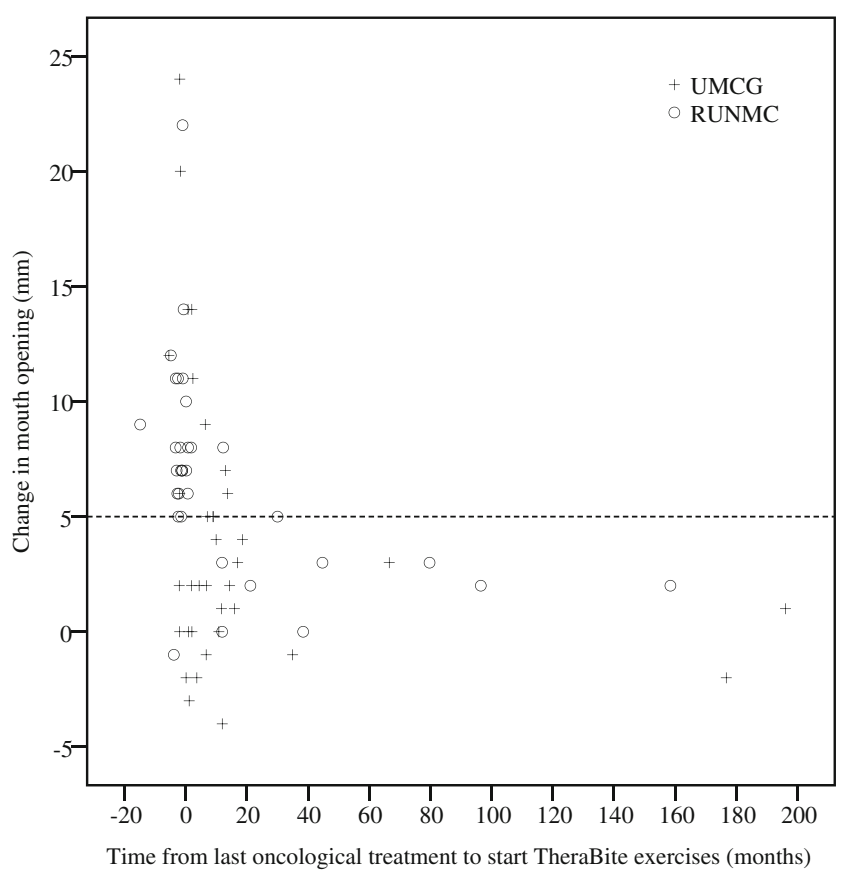

Fig. 1 Increase in mouth opening plotted against time from oncological treatment to start exercises
(SD 8.1) [21]. The difference in gain in mouth opening between that and our study might be explained by differences in population. Additionally, patients in the study of Cohen et al. began their exercises within 6 weeks after surgery and had a less severely restricted mouth opening prior to the exercises (initial mouth opening of $40 \mathrm{~mm}$ versus $22.0 \mathrm{~mm}$ in our population). Moreover, only two of the seven patients ( 29 versus $78 \%$ in our population) were treated with radiotherapy before the exercise therapy started [21].

Mouth opening increased with a comparable amount of millimeters (6.2 mm, SD 3.4) when trismus was treated with another type of mechanical stretching device, the DTS. In that study, the interval between oncological treatment and initiation of exercise therapy was approximately 4 years [23]. In a preliminary report and in a case series, mouth opening increased on average with 11.0 and $13.6 \mathrm{~mm}$, respectively, after exercises with the DTS $[18,22]$.

Regression to the mean might explain the increase in mouth opening in the current study, indicating that a patient seeks help at the peak of the burden of symptoms (a maximally restricted mouth opening). Restricted mouth opening shows a random variation over time. Due to this variation, it can be expected that mouth opening increase after some time independent of any intervention. However, in head and neck cancer patients, who have been treated with radiotherapy, mouth opening decreases over time [27]. Thus, we assume that regression to the mean is not a likely explanation for the increase in mouth opening and that the increase can be attributed to the exercises.

The fracture of a titanium reconstruction plates during TheraBite exercises is an adverse effect that can be explained by non-union of the fibula to the mandible, by the recurrence of carcinoma, or by excessive stretching forces. When prescribing exercises with a TheraBite in cases of bony reconstructions of the mandible, exercise forces should be limited until consolidation of the reconstruction is completed.

Since it is difficult to treat a head and neck oncologyrelated trismus, more attention should be paid to prevent trismus by applying intensity modulated radiotherapy when possible or by starting (TheraBite) exercises during or 
immediately after oncological treatment [28]. However, exercises may not be effective in preventing a decrease in mouth opening [29]. In our study, patients were encouraged to continue exercise therapy until no further improvement in mouth opening was achievable. Compliance to exercise therapy is dependent on internal motivation and the perceived effect of exercises. Perceiving no treatment effect, reaching the exercise goal, and an insufficient opening range of the TheraBite have a negative effect on adherence [4]. None of the patients, who started with the exercises after 3 years, reached more gain in mouth opening than $3 \mathrm{~mm}$. Therefore, the value of this type of exercises after an interval of 3 years is limited (Fig. 1).

In this study, no patients were excluded from a TheraBite for financial reasons. However, based on the outcome of this study, not every patient should straightforwardly be treated with exercise therapy with a TheraBite because of the high costs of the apparatus and the comparable effects of the much cheaper conventional exercise therapy. To the best of our knowledge this study included the largest sample of patients treated for a head and neck oncology-related trismus using a TheraBite. The overall absolute increase in mouth opening using a TheraBite found in this study was comparable to conventional exercise therapy [19].Patients should be informed about the results they can expect of TheraBite exercise therapy.

\section{Study limitations}

Limitations of the current study are lack of a control group, its primarily retrospective design, lack of the assessment of compliance, and a non-blinded assessment of mouth opening that may have led to an over- or underestimation of the effect of TheraBite exercises. Because most data were gathered before the intention of the study was clear, we assume a random error in measuring mouth opening. Different persons in two medical centers measured mouth opening, but mouth opening can be measured reliably; therefore, we assume that this had no systematic effect on the outcome of this study [25]. Another limitation is that all patients who were referred for TheraBite exercises were included in the study, resulting in considerable heterogeneity in patient population and oncological treatment characteristics. However, this heterogeneity reflects clinical practice and therefore enhances external validity. Finally, a limitation of the study is that the effect TheraBite exercise therapy only was measured as mouth opening in millimeters. Mandibular functioning or quality of life was not assessed; however, the TheraBite is primarily developed for increasing mouth opening.

Further research is needed to compare the TheraBite with the DTS in a randomized controlled trial. The costs of a DTS are about five times higher than those of a TheraBite. Cost effectiveness of both jaw-stretching devices should be analyzed. Effects of the exercises should ideally be measured in millimeters, in mandibular functioning, and in quality of life.

\section{Conclusions}

Mouth opening increased averagely with $5.4 \mathrm{~mm}$ after exercises with the TheraBite. The results indicate that the odds of an increase in mouth opening of $5 \mathrm{~mm}$ or more reduces if the time from oncological treatment to start exercises lengthens, corrected for the effect of medical center.

Open Access This article is distributed under the terms of the Creative Commons Attribution Noncommercial License which permits any noncommercial use, distribution, and reproduction in any medium, provided the original author(s) and the source are credited.

\section{References}

1. Dijkstra PU, Huisman PM, Roodenburg JL (2006) Criteria for trismus in head and neck oncology. Int J Oral Maxillofac Surg 35:337-342

2. Johnson J, van As-Brooks CJ, Fagerberg-Mohlin B, Finizia C (2010) Trismus in head and neck cancer patients in Sweden: incidence and risk factors. Med Sci Monit 16:CR278-CR282

3. van der Molen L, van Rossum MA, Ackerstaff AH, Smeele LE, Rasch CR, Hilgers FJ (2009) Pretreatment organ function in patients with advanced head and neck cancer: clinical outcome measures and patients' views. BMC Ear Nose Throat Disord 9:10

4. Melchers LJ, Van Weert E, Beurskens CH, Reintsema H, Slagter AP, Roodenburg JL, Dijkstra PU (2009) Exercise adherence in patients with trismus due to head and neck oncology: a qualitative study into the use of the Therabite. Int J Oral Maxillofac Surg 38:947-954

5. Scott B, Butterworth C, Lowe D, Rogers SN (2008) Factors associated with restricted mouth opening and its relationship to health-related quality of life in patients attending a Maxillofacial Oncology clinic. Oral Oncol 44:430-438

6. Bensadoun RJ, Riesenbeck D, Lockhart PB, Elting LS, Spijkervet FK, Brennan MT, Trismus Section, Oral Care Study Group, Multinational Association for Supportive Care in Cancer (MASCC)/ International Society of Oral Oncology (ISOO) (2010) A systematic review of trismus induced by cancer therapies in head and neck cancer patients. Support Care Cancer 18:1033-1038

7. Louise Kent M, Brennan MT, Noll JL, Fox PC, Burri SH, Hunter JC, Lockhart PB (2008) Radiation-induced trismus in head and neck cancer patients. Support Care Cancer 16:305-309

8. Weber C, Dommerich S, Pau HW, Kramp B (2010) Limited mouth opening after primary therapy of head and neck cancer. Oral Maxillofac Surg 14:169-173

9. Kamstra JI, Jager-Wittenaar H, Dijkstra PU, Huisman PM, van Oort RP, van der Laan BF, Roodenburg JL (2011) Oral symptoms and functional outcome related to oral and oropharyngeal cancer. Support Care Cancer 19:1327-1333

10. Abdel-Galil K, Anand R, Pratt C, Oeppen B, Brennan P (2007) Trismus: an unconventional approach to treatment. Br J Oral Maxillofac Surg 45:339-340 
11. Alexander SA, Renner RP (1989) Increasing occlusal vertical dimension with an orthodontic 'clothes pin appliance'. A clinical report. J Prosthet Dent 62:1-3

12. Lund TW, Cohen JI (1993) Trismus appliances and indications for use. Quintessence Int 24:275-279

13. Rahn AO, Drone JB (1967) Dental aspects of the problems, care, and treatment of the irradiated oral cancer patient. J Am Dent Assoc 74:957-966

14. Ziccardi VB, Ochs MW, Braun TW (1995) Intraoperatively fabricated bite block in the management of scar contracture hypomobility. Oral Surg Oral Med Oral Pathol Oral Radiol Endod 80:34-35

15. Brown KE (1968) Dynamic opening device for mandibular trismus. J Prosthet Dent 20:438-442

16. Fonseca EP (1969) Treatment of maxillomandibular constrictions. J Prosthet Dent 22:652-656

17. Hartl DM, Cohen M, Julieron M, Marandas P, Janot F, Bourhis J (2008) Botulinum toxin for radiation-induced facial pain and trismus. Otolaryngol Head Neck Surg 138:459-463

18. Stubblefield MD, Manfield L, Riedel ER (2010) A preliminary report on the efficacy of a dynamic jaw opening device (dynasplint trismus system) as part of the multimodal treatment of trismus in patients with head and neck cancer. Arch Phys Med Rehabil 91:1278-1282

19. Dijkstra PU, Sterken MW, Pater R, Spijkervet FK, Roodenburg JL (2007) Exercise therapy for trismus in head and neck cancer. Oral Oncol 43:389-394

20. Buchbinder D, Currivan RB, Kaplan AJ, Urken ML (1993) Mobilization regimens for the prevention of jaw hypomobility in the radiated patient: a comparison of three techniques. J Oral Maxillofac Surg 51:863-867

21. Cohen EG, Deschler DG, Walsh K, Hayden RE (2005) Early use of a mechanical stretching device to improve mandibular mobility after composite resection: a pilot study. Arch Phys Med Rehabil 86:1416-1419

22. Shulman DH, Shipman B, Willis FB (2008) Treating trismus with dynamic splinting: a cohort, case series. Adv Ther 25:9-16

23. Baranano CF, Rosenthal EL, Morgan BA, McColloch NL, Magnuson JS (2011) Dynasplint for the management of trismus after treatment of upper aerodigestive tract cancer: a retrospective study. Ear Nose Throat J 90:584-590

24. Sobin LH, Gospodarowicz MK, Wittekind C (2009) TNM Classification of Malignant Tumours:336

25. Jager-Wittenaar H, Dijkstra PU, Vissink A, van Oort RP, Roodenburg JL (2009) Variation in repeated mouth-opening measurements in head and neck cancer patients with and without trismus. Int J Oral Maxillofac Surg 38:26-30

26. Scott B, D'Souza J, Perinparajah N, Lowe D, Rogers SN (2011) Longitudinal evaluation of restricted mouth opening (trismus) in patients following primary surgery for oral and oropharyngeal squamous cell carcinoma. Br J Oral Maxillofac Surg 49:106-111

27. Wang CJ, Huang EY, Hsu HC, Chen HC, Fang FM, Hsiung CY (2005) The degree and time-course assessment of radiationinduced trismus occurring after radiotherapy for nasopharyngeal cancer. Laryngoscope 115:1458-1460

28. Chao KS, Deasy JO, Markman J, Haynie J, Perez CA, Purdy JA, Low DA (2001) A prospective study of salivary function sparing in patients with head-and-neck cancers receiving intensitymodulated or three-dimensional radiation therapy: initial results. Int J Radiat Oncol Biol Phys 49:907-916

29. Grandi G, Silva ML, Streit C, Wagner JC (2007) A mobilization regimen to prevent mandibular hypomobility in irradiated patients: an analysis and comparison of two techniques. Med Oral Patol Oral Cir Bucal 12:E105-E109 